\title{
SECURITY ON ATM NETWORKS
}

\author{
Stelios Karanastasis and Ahmed Patel \\ Department of Computer Science, University College Dublin, \\ Belfield Campus, Dublin 4, Ireland, \\ Tel: ++353 I 7062488, \\ stkara@aegean.gr,apatel@net-cs.ucd.ie
}

\begin{abstract}
This paper discusses the ATM security problems, requirements, implementation issues and challenges. It also presents a survey of the existing solutions aiming to secure the data transferred over an ATM network. Different solutions are presented analysed and compared. Details are given about the security services offered their placement within the ATM Reference Model and the techniques to provide synchronisation and dynamic key change during user data exchange.
\end{abstract}

\section{INTRODUCTION}

In recent years, security has been more and more significant in network environment with the emergence of the internetworking technology. The internetworking technology can provide the communication channels across networks so that, machines in different networks can talk to each other. However, the internetworking communication will be exposed to all kinds of attacks in such an open environment. Most of the network technologies, without integrating with security mechanism originally, have to be redesigned to provide some security services. ATM is one of those technologies.

The Asynchronous Transfer Mode (ATM) forms the basics for many broadband networks, and it forms part of the foundation for B-ISDN networks. ATM uses multiplexing, switching and segmentation/reassemble operations to support a high-speed transport network. It was designed to make Broadband-ISDN a reality. B-ISDN was created conceptually as just 
an extension of ISDN so it functions as a communication network that can provide integrated broadband services such as high speed data services, video phone, video conferencing, CATV services along with traditional ISDN services such as phone an telex. In order to have these services, an interface between the ATM layer and higher layers was necessary. The ATM adaptation layer provides this service. Its main purpose is to resolve any disparity between a service required by the user and services available at the ATM layer. It lies between the ATM layer and the higher layers of B-ISDN protocol reference model, as can be seen in Figure 1 [I.321]. The user plane is responsible for users data exchange; the control plane monitors signalling information; and the management plane maintains the network operational.

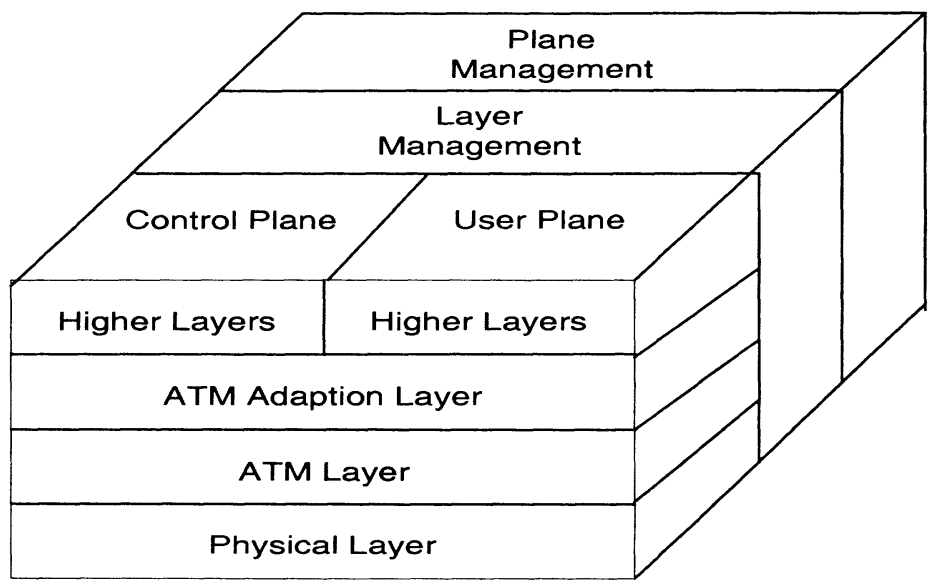

Figure 1. B-ISDN reference model and ATM

In fact, issues in ATM security have not gained enough attention until 1995, when a group within ATM Forum was established to address the security issues. Therefore, compared with other security area, ATM security is still in its beginning. Security in ATM networks is not only concerned with the user data but all the protocol control data as well. The later consists of both management and signalling information which needs to be made secure so that an attacker/intruder can't change pertinent or significant data in order to divert or destroy the proper functioning of the ATM network service (e.g. converting VCs). With no security services being currently offered in ATM specifications, communications passed over ATM networks will suffer a lot of threats. These threats are examined in section 2 . Then we discuss the requirements of ATM security followed by the implementation of ATM security in the ATM architecture according to the standards 
proposed by the ATM Forum. In section 5, we describe, analyse and compare the different approaches of securing ATM networks. Finally in section 6 we discusses some major challenges in ATM security.

\section{THREATS TO ATM NETWORKS}

As other networks, ATM networks will suffer a lot of threats [Alles95], [Chuang96], [Deng95], [TayFin95], [Hanson95]. Typical ones are eavesdropping, spoofing, service denial, traffic analysis, VC stealing etc. Note that VC stealing is a new threats for networks since is only applies to ATM networks.

Eavesdropping: Eavesdropping refers to the threat that the attacker connects or taps into the transmission media and gain unauthorised access to the data. It is one of the most common attacks to the network. Although a hacker has to be familiar with the communication technology and relevant protocols operating at the tapping point, information that is widely available in academic environment. As the technology become matured, standards will be established and the technology will be well known, nothing will be protected by keeping the document secret [Hanson95].

Spoofing: Spoofing attack means that an attacker tries to impersonate another user to the third party therefore can get access to resources belonging to the victim to take advantages or just destroy them. Spoofing might need special tools to manipulate the protocol data unit and requires special access permissions. However, since a network will be connected to many untrusted networks via the Internet, it's almost impossible to prevent a hacker from getting this access permission or even trace the people with this particular access permission. ATM is being implemented in public domain. Therefore, it is subject to this kind of attack also.

Service Denial: ATM is a connection-oriented technique. A connection, which is called Virtual Circuit(VC) in ATM, is managed by a set of signals. VC is established by SETUP signals and can be disconnected by RELEASE or DROP PARTY signals. If an attacker sends RELEASE or DROP PARTY signal to any intermediate switch on the way of a VC, then the VC will be disconnected. By sending these signals frequently, the attacker can greatly disturb the communication between one user to another, therefore will disable the Quality of Service (QoS) in ATM. Combining this technique with other tricks like eavesdropping, the attacker can even completely block one user from another. 
Traffic Analysis: Traffic analysis consists of an attack whereby the communication channel is tapped and statistical information about the data traffic is accumulated. Specifically, the volume, timing, source and destination addresses of communication data can be collected by the attacker. This information in conjunction with knowledge of the routing tables contained in the network switching devices (ATM switches will work with routing information which is unencrypted unless specific efforts are made to hide it) can be a very useful tool for the attacker. Thus extra provision needs to be made for protection against traffic analysis attack Another related threat is called convert channels. In this technique, the attacker can encode the information in the timing and volume of data, VCI, or even session key to release information to another people without being monitored. Normally, these two attacks aren't easy to implement. However, when ATM is used in an environment requiring stringent security, it might happen. [TayFin95]

Stealing of VCs: Switches are forwarding cells based on the VCI((Virtual Channel Identifier) or VPI(Virtual Path Identifier) in the cell header. If an attack manage to change these values at the end point switches of an ATM connection then he will be able to forward his cell via another connections that has for example better Quality of Service. The switches in the middle will not notice these changes and will switch the "faked" cells just like the authentic cells. In public packet-switching network, the attacker won't gain too much by this trick. However, in ATM network, if quality of service is guaranteed, then he can gain a lot by stealing a higher quality channel which he is not entitled to use according to the access control policy. The possibility that the end-point switches will compromise would be pretty low, if the ATM network is owned by one organisation. However, when we consider ATM internetworking, in which case cells will travel through different ATM networks, it will be very easy for two switches to compromise [Alles95].

A threat is a potential violation of a security objective. Summarising we can distinguish three kinds of threats [ATMForum98]:

- An accidental threat, where the origin of the threat does not involve any malicious intent

- An administrative threat, where the threat arises from a lack of administration of security

- Intentional threats, where the threat involves a malicious entity, which may attack either the communication itself or network resources. 


\section{REQUIREMENTS OF ATM SECURITY SYSTEM}

To build an ATM security system, the first thing we should do is to identify the requirements of securing communication over ATM. Recently, ATM Forum Security Working Group proposes a draft of Security Framework for ATM [ATMForum98] to address the basic requirements for ATM security. It is based on the analysis of the objectives from the customer side, operator side and public community side, and identifies the main security objectives for ATM security:

- Confidentiality

- Integrity

- Accountability

- Availability

Confidentiality is not only required to keep the data from unauthorised access, but also can guarantee the correctness of distribution of symmetric key. Integrity can be viewed as some kind of authentication, which means the data should be the original one sent by the one who claims it. Accountability means that all ATM network service invocations and network management activities should accountable. And any entity should be responsible for the actions it initiates. Accountability includes both authentication and non-repudiation. It is extremely important for operators to manage the system and bill the services. Availability means all legitimate entities should be able to access to ATM facilities correctly, no service denial should happen. That is important for QoS operation.

- According to these main objectives, the ATMForum proposes the principal functions, which an ATM security system should provide [ATMForum98]:

- Verification of Identities: Security system should be able to establish and verify the claimed identity of any actor in an ATM network.

- Controlled Access and Authorisation: The actors should not be able to gain access to information or resources if they are not authorised to.

- Protection of Confidentiality: Stored and communicated data should be confidential.

- Protection of Data Integrity: The security system should guarantee the integrity of the stored and communicated data.

- Strong Accountability: An entity can not deny the responsibility of its performed actions as well as their effects.

- Activities Logging: The security system should support the capability to retrieve information about security activities in the Network 
Elements with the possibility of tracing this information to individuals or entities.

- Alarm reporting: The security system should be able to generate alarm notification about certain adjustable and selective security related events.

- Audit: When violations of security happen, the system should be able to analyse the logged data relevant to security.

- Security Recovery: The security system should be able recover from successful or attempted breaches of security.

- Security Management: The security system should be able to manage the security services derived from the above requirements.

Among the ten requirements, the last two wont provide security services. However, they are necessary to support the maintenance of security services. If the security system can not be recovered from attacks and can not provide security services any more, then the system wont be secure after these attacks. On the other hand, security services and the information about security have to be managed securely. They are the foundations of the security system. In the following section we will present the existing solutions aiming to secure communications over ATM networks.

\section{IMPLEMENTING SECURITY SERVICES ON ATM}

In order to understand correctly the different approaches on ATM security, it would be useful enough to discuss the ATM security scope and how to place the security services in the ATM architecture.

The ATM architecture includes three planes, (see Figure 1) the User plane to exchange user data, the control plane to monitor signalling information and the management plane to maintain the network operational. In particular the management plane includes the PNNI functions related to establishment of a routing infrastructure. User and control planes communicate over the ATM network through reserved virtual channels. The management plane uses the same channel as the user plane to exchange Operation And Maintenance (OAM) cells. All information is exchanged over the network in the form of 53-byte ATM cells. The ATM reference model also includes three lower layers, a physical layer mainly responsible for information transportation, an ATM layer mainly in charge of multiplexing and switching functions and an ATM Adaptation Layer (AAL) whose main function is to adapt services needs to ATM streams by 
performing the assembly/disassembly of the cells. It is obvious that to implement the security requirements, mentioned before, in ATM networks, all the three planes and the ATM layer have to be included into the scope (see Figure 2).

\subsection{Placement of ATM Security Services}

As can be seen in Figure 2, user plane is the plane that directly interact with user. Therefore to meet the user's security objectives, user plane has to provide security services like access control, authentication, data confidentiality and integrity [GriKa96]. Other services like key exchange, certification infrastructure and negotiation of security options might be useful to meet the variety of the customers' requirements. Therefore they also should be supported by user plane [ATMForum97]. Note that to provide different security services options is important because of the various traffic classes in ATM network. Different connections have different security requirements. User plane security services have to provide enough flexibility to meet these requirements.

In ATM Control plane will configure the network to provide communication channel for a user. Control plane can interact with the switching table, or to manage the virtual channel (Figure 2). Several attacks mentioned in the previous section are relative to control plane. Therefore, it is very important to secure control plane. The key point to secure control plane may be to provide authentication and confidentiality of signal [ATMForum97]. If the message recipient or even the third party can verify the source of this message, then denial of service attack can not happen. And control plane authentication could also be used to provide the auditing information for accurate billing, which should be immune to repudiation.

Management plane security is also important. If we want a stiff management plane security scheme then we should at least consider the following items: Bootstrapping security, authenticated neighbour discovery, the Interim Local Management Interface security and permanent virtual circuit security [Chuang96]. And in security framework, we have to provide security recovery and security management. The major parts of these two requirements seem have to be implemented in management plane. 


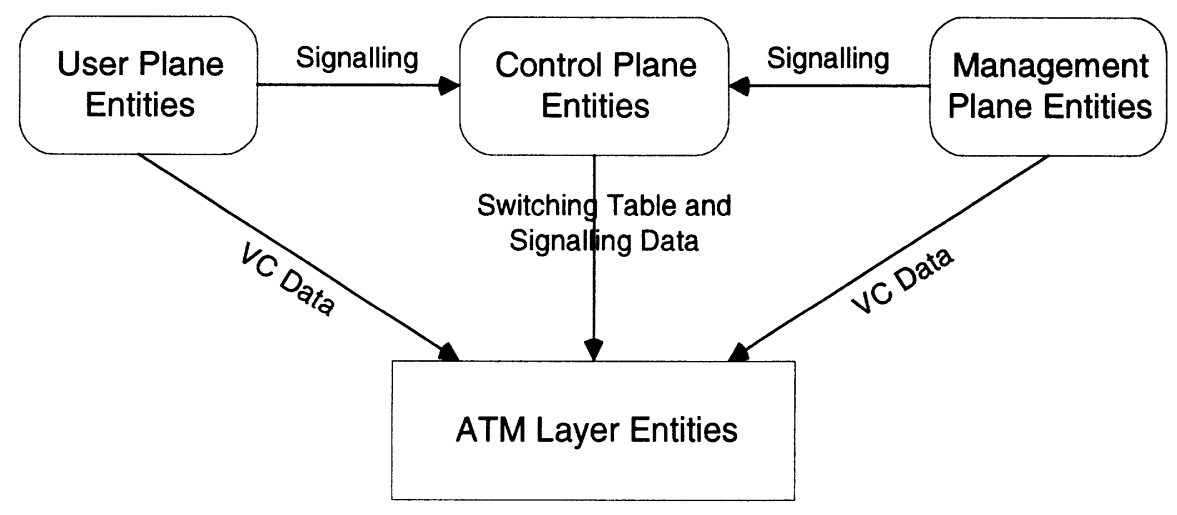

Figure 2. ATM Entities Interaction Model

Since all data have to be transmitted through ATM layer, the security of ATM layer is extremely important. Authentication, confidentiality and integrity are also required in the ATM layer. ATM layer security has to be implemented on end-to-end(ATM endpoint to ATM endpoint), edge to edge (border ATM switch to border ATM switch) and ATM end point to switches basis. Here, data integrity is a tricky thing. Since the switches can see and forward ATM cells, data integrity for edge-to-edge and ATM endpoint to switches is better to be implemented on a per-ATM-cell basis. That means we have to include a signature to each cell. That will introduce a unwanted overhead. Therefore, integrity should be provided on an endpoint to endpoint basis [ATMForum95].

Endpoint to endpoint security in ATM layer is different from the security in higher layer. An ATM layer connection is not the same as a higher layer connection. Therefore although a ATM connection is authenticated and secure, higher layer connection still should be authenticated and protected. This is necessary especially when a connection is set up for a legacy network, which is connected to a ATM switch. In this case, the connection will be shared by all hosts on the legacy network. Actually, the security issue of this scenario is still untouched yet.

\section{SOLUTIONS ON ATM SECURITY}

Solutions to secure ATM communications are discussed in [Chuang96], [Deng95], [SteHilBy95] and [ATMForum97]. As securing an ATM communication is based on firstly negotiating a security context and secondly applying negotiated security services on user data exchanges as 
well as protocol control-data, these solutions' presentation is divided into two corresponding subsections. The following table, Table 1, is summarising the security services offered by all the ATM security solutions discussed in the following paragraphs.

Table 1. Security services offered by ATM security solutions

\begin{tabular}{|c|c|c|c|c|}
\hline & Stevenson et al & Deng et al & Chuang & ATM Forum \\
\hline $\begin{array}{l}\text { Security Services } \\
\text { established } \\
\text { through } \\
\text { signalling }\end{array}$ & & $\begin{array}{l}A, K E, R D, \\
S N: I+C\end{array}$ & $\begin{array}{l}\text { A,KE,RD,SN: } \\
\text { I+C+KE for } \\
\text { all signalling } \\
\text { messages }\end{array}$ & $\begin{array}{l}\mathrm{A}, \mathrm{I}, \mathrm{AC}, \mathrm{KE}, \\
\mathrm{RD}, \mathrm{SN}: \mathrm{I}+\mathrm{C}+ \\
\mathrm{KE}+\mathrm{A} \text { for all } \\
\text { signalling } \\
\text { messages }\end{array}$ \\
\hline $\begin{array}{l}\text { Security Services } \\
\text { established } \\
\text { through } \\
\text { management cells }\end{array}$ & & & & $\mathrm{KE}$ \\
\hline $\begin{array}{l}\text { Security Services } \\
\text { established } \\
\text { through the } \\
\text { auxiliary channel }\end{array}$ & $\mathrm{EC}, \mathrm{A}, \mathrm{RD}$ & & & $\mathrm{SN}, \mathrm{A}, \mathrm{KE}$ \\
\hline User data services & $\begin{array}{l}\text { C within ATM, } \\
\text { S with OAM or } \\
\text { user data cells }\end{array}$ & $\begin{array}{l}\mathrm{C}, \mathrm{I} \text { and }(\mathrm{RD}) \\
\text { within } \mathrm{AAL}, \mathrm{S} \\
\text { done for each } \\
\text { DPL-PDU, KE }\end{array}$ & $\begin{array}{l}\text { C within } \\
\text { ATM, I,A } \\
\text { within AAL, } \\
\text { S within AAL } \\
\text { with a } \\
\text { cryptotag }\end{array}$ & $\begin{array}{l}\text { C rather } \\
\text { within ATM, } \\
\text { I,A and (RD) } \\
\text { within AAL, } \\
\text { S with OAM } \\
\text { cells }\end{array}$ \\
\hline
\end{tabular}

\section{Notations :}

\begin{tabular}{|c|c|c|c|}
\hline A & Authentication & $\mathrm{KE}$ & Keys Exchange \\
\hline $\mathrm{AC}$ & Access Control & $\mathrm{P}$ & Padding \\
\hline $\mathrm{C}$ & Confidentiality & $\mathrm{RD}$ & Replay Detection \\
\hline I & Integrity & $\mathrm{SN}: \mathrm{XX}$ & Negotiation of service $\mathrm{XX}$ \\
\hline$(\mathrm{XX})$ & Optional service XX & & \\
\hline
\end{tabular}

\subsection{Security services negotiation}

ATM security solutions differ in that security services negotiation is done:

- During connection set up within control operations [ATMForum97], [Chuang96]

- along the connection within management operations [ATM Forum97]

- over an auxiliary channel dedicated to security operations [SteHilBy95], [ATMForum97] 
Note that ATM Forum's solution uses all these schemes to exchange security information.

\subsubsection{Negotiation trough signalling information}

This scheme consists in inserting security information (session keys, security mechanisms and authenticator) into signalling messages such as SET UP and CONNECTS within one or more Information Elements (IEs).

The ATM Forum [ATMForum97] limits signalling support to the authentication and access control services. Other security services are realised by other means presented in the following sections. The ATM Forum defines a new IE, which allows to authenticate the source of a signalling message. Authentication may be done end-to-end (between two end-entities) or hop-by-hop (between switches and end-entities) in connection set up messages (SET UP and CONNECT) and other signalling messages such as connection release. Additionally to the authentication service, the IE ensures integrity of part or all the signalling message and replay detection with the Sequence number and Timestamp fields. One access control specific IE is also defined to specify the sensitivity level of user data that need to be exchanged over the connection so that, for instance, the path selected over the network uses only links with appropriate sensitivity level.

Deng's solution [Deng95] defines twelve new IEs - six for each SET UP and CONNECT message - which allow different entities to authenticate mutually, exchange unidirectional session keys (2 IEs) and negotiate a security context (4 IEs). Only confidentiality and integrity services to be applied on user data may be negotiated. Authentication and keys exchange are based on X509 two way authentication protocol. This scheme is rigid since the authentication protocol is imposed. Moreover using as many IEs as security parameters makes this scheme clear but not optimised in terms of modifications implied within UNI specifications [ATMForum96].

Chuang [Chuang96] developed its solution within the MSN-CMA architecture (Multi Service Network Connection Management Architecture) which is based on servers supervising all ATM network's management and control operations. Contrary to the ATM Forum and the ITU-T, a connection set up requires the exchange of three signalling messages instead of two so that two way and three way authentication protocols may be chosen for that solution to authenticate and exchange session keys. This three way handshake connection set up enables to negotiate, amongst others, confidentiality, integrity and keys exchange mechanisms to be used for 
securing user data exchanges. Also Chuang suggests that security parameters renegotiations be done through signalling exchanges.

\subsubsection{Negotiation through management information}

This scheme consists in injecting security information in-band within the user data channel by the means of OAM cells. The only solution applied so far in this area is from the ATM Forum [ATMForum97]. The ATM Forum uses such a scheme when security information requires synchronisation with the user data cells stream, for instance to exchange new session keys during a connection. It also defines new OAM cells dedicated to security in order not to confuse them with ordinary OAM cells and suggests to encapsulate security messages into the 45-byte OAM cells' function specific field. To protect security messages exchanges, protocols based on symmetric or asymmetric algorithms are specified.

\subsubsection{Negotiation through an auxiliary channel}

Auxiliary channel notion is used here when security information is exchanged in the form of user data cells. Stevenson [SteHilBy95] suggests to negotiate session keys at connection set up within the first data exchanged. The solution that the ATM Forum presents is used in conjunction with the solution to negotiate security parameters (security mechanisms). It may also be used to do mutual authentication during connection or at connection set up (in case a three-way authentication is required additionally to the twoway authentication realised within signalling messages). This solution applies in a proxies context, however contrary to the Stevenson's solution, the auxiliary channel is a specific channel entirely dedicated to security parameters exchanges.

\subsubsection{Comparison of the solutions}

The solutions described above can be compared in terms of modifications required within the ATM Forum's UNI 3.1 specifications [ATMForum94]. Whereas Deng, respectively the ATM Forum, introduce twelve new IEs, respectively two new IEs and security OAM cells, Stevenson and Chuang require no UNI modifications since modifications are transparent for the ATM network. Stevenson modifies only the connection content and Chuang modifies proceedings within stations and servers (software). 
All the solutions though have some drawbacks. The ATM Forum's (negotiation through signalling information) and Deng's solutions expect intermediary public network equipment to transparently pass security information elements which may be used as covert channel (to pass data for free in case signalling is free). As a consequence, operators are reluctant to introduce new security IEs in signalling messages.

Both in-band and auxiliary channel schemes include flaws. The former is unsuitable for unidirectional communications and real-time applications. The latter is costly in terms of connections set up (in ATM Forum 's implementation through an auxiliary channel) or does not enable security parameters renegotiations during a connection (in Stevenson's implementation).

The environment of Chuang's solution is not compliant to ATM Forum and ITU-T's philosophy where management and control operations are distributed among network equipment so that his solution is not applicable to the current ATM standard environment.

\subsection{Secure user data exchange}

User data exchanges may be protected with the security services negotiated in the previous section. Solutions differ in that user data encryption is performed:

- at the ATM layer [SteHilBy95], [Chuang96], [AtmForum97]

- within the AAL layer [Deng95]

\subsubsection{Confidentiality at the ATM layer}

This scheme consists in encrypting user data on the cell-by-cell basis. However encryption algorithms are more or less error extension sensitive. That is, cell loss occurrence may cause for instance at most one 64-byte block loss when encrypted with DES ECB (Electronic Code Book) and much more data loss when encrypted with DES CBC (Cipher Block Chaining), since in the ECB mode, blocks of data are encrypted independently whereas in the CBC mode, an encrypted block of data is dependent on the plain text block that generated it but also all the previous encrypted blocks. To avoid too many losses to occur, encrypting/decrypting devices need to be frequently resynchronised. Hereafter each solution's synchronisation mechanism is specified.

Stevenson [SteHilBy95] defines cryptographic units, which encrypt ATM cells with one session key per connection. He studies the "key agility" 
problem, i.e. the problem of switching keys quickly enough so that ATM cells streams from various connections can be encrypted independently. He also solves the synchronisation problem by injecting synchronisation information in-band within OAM cells or user data cells.

Chuang [Chuang96] defines a "cryptonode" device encrypting user data at the ATM layer with one session key per connection. Synchronisation is performed at the AAL layer by injecting AAL5 PDU tokens including new keys numbers and initialisation vectors (IV) that should be used for decrypting the next block of data. Therefore when a key or IV change is detected at the decryption device (two consecutive tokens contain different keys or IVs), an interrupt is generated to the ATM layer decryption process, so that cells decryption is halted and temporarily controlled by the AAL layer for keys or IV update. In such a scheme, a problem may occur since synchronisation is realised at a different layer than encryption. Indeed when a synchronisation token is received mixed up with user data cells of the same connection, during the token processing at the AAL layer, many user data cells may be decrypted at the ATM layer with the old security context (old keys or IVs).

Additionally to synchronisation, tokens support user data integrity/authentication by the means of a signature appended to tokens together with integrity keys and IV.

The ATM Forum [ATMForum98] supports user data confidentiality and integrity (with two independent keys) and optionally reordering/replay detection. Confidentiality may be realised end-to-end or switch-to-switch whereas integrity is only ensured end-to-end. Indeed, the confidentiality service is provided at the ATM layer but confidentiality at the AAL layer is also suggested for the end-to-end scenario. The integrity and optional reordering/replay detection are supported at the AAL layer by the means of fields appended to the AAL SDU to protect: a signature computed over the AAL SDU, a timestamp and a sequence number. As mentioned before, synchronisation together with keys negotiation are realised in-band within OAM cells.

\subsubsection{Confidentiality within the AAL layer}

User data encryption is provided within the AAL layer prior to their segmentation into cells. Deng [Deng95] defines an additional layer called DPL (Data Protection Layer) which is placed between two AAL sublayers and supports confidentiality and integrity services and optional reordering. DPL SDUs are independently encrypted and signed with the keys negotiated 
at connection set up and the Initialisation Vector (IV) specified within the DPL SDU's clear header. As a consequence no synchronisation is needed.

During connection the DPL layer may update session keys by halting data transmission and sending repeatedly one DPL SDU containing a new session key (encrypted with its private key) until reception of a positive acknowledgement. This scheme appears unsuitable for unidirectional communications and real-time applications and is unsecured since no strong authentication protocols are used to exchange session keys.

\subsubsection{Comparing the Solutions}

In this section we compare the above implementations in terms of services supported. Stevenson's solution only supports the confidentiality service whereas all other solutions support confidentiality and integrity services. At this point we should note that the integrity service when offered is always placed at the AAL layer in order to avoid additional cells segmentations whereas the confidentiality service is placed at the ATM or AAL layer.

Encrypting user data at the ATM layer brings simplicity and performances improvements since encryption is realised on fixed size cells and within hardware. Benefits result also from encrypting user data at the AAL layer. Modifications within the ATM layered model are restricted to the AAL layer. Because of encryption realised on long size block of data (up to 65535 bytes for DPL SDUs), no key agility problems arise. In Deng's solution, only session keys change requires synchronisation with the user data flow and synchronisation is easier to realise than in Chuang's solution since synchronisation and encryption are done within the same layer like with ATM Forum and Stevenson's solutions. However placing confidentiality at the AAL layer is less reliable than at the ATM layer since DPL PDUs and AAL PDUs' headers remain in clear.

It should be pointed out that, contrary to ATM Forum and Chuang's solutions, the integrity and confidentiality services are not independent in Deng's solution since both of them use the same key. This results in algorithms choice restrictions and a weaker security level since in case the used key is broken, user data exchanged may be decrypted but also corrupted user data may be generated and signed. 


\section{MAJOR CHALLENGES IN ATM SECURITY}

At the first glimpse, ATM security should not be too difficult to implement since we have a various security practices in other fields. However, ATM security is very difficult to implement. ATM switch is a high-speed cell multiplexer and ATM network is a connection-oriented network. These properties will bring some unique problems when we try to secure the ATM communication. In this section we will present some challenges in implementation of ATM security mechanism, most of them can be seen in [Chuang96].

It is clear that encryption is the basis for a lot of security problems. In ATM security, encryption will happen in two level. One level is the application level. There wont be any problem with it since we can apply any security mechanism. Another level is the ATM layer level. In this level, we are going to deploy security mechanism to a switch. Since switch see and forward only cells, we have to apply security mechanism to a cell. That can not be totally avoided in ATM network. For example, if we want to provide integrity and confidentiality services to the cell header, or if we want to protect the signals, then we have to do encryption/decryption a cell in each switch. That will bring a series adverse effects.

The first challenge in securing ATM network is how to find a cryptographic mechanism to match the high communication speed of a switch. Cryptography is used to provide confidentiality, authentication, and even integrity service for a security system. Unfortunately, most traditional cryptographic mechanisms operate within $10 \mathrm{Mbps}$ when implemented in software or hundred Mbps when implemented in hardware. This speed is impossible to meet the speed of a switch, which normally will operate at hundreds of Mbps up to Gbps. Although [Eberle92] had proposed an implementation of DES, which can operate at Gbps, it takes a big time overhead to warm up when the session key changes. Due to key agility requirement we shall introduce, it's not a good solution to that problem. From here we can see why Phase I Security Specification [ATMForum97] hasn't addressed how to protect the cell header.

Another issue will rule out a lot of traditional cryptographic mechanisms. ATM cell payload is 48 bytes. Therefore any block cipher with block size more than 384 bits can not be applied to encrypt a cell. Even the block size of a cipher is smaller than 384 bits, the alignment of the cell and cipher block also affect the choice of a cryptographic algorithm. Phase I Security Specification will encounter this problem since the data confidentiality in user plane is on per cell basis. An alternate for block ciphers is stream 
ciphers. However, this solution will suffer from the problem of resynchronisation. If a cell is lost during the transmission, then when the receiver receives and decrypt the cell sequence, the data will look like garbage.

Even we can find a cryptographic mechanism, which can meet the above requirements, high speed in ATM can introduce difficulties in key management. With such a high speed, ATM can transfer a high volume of data in a short time. Assume an ATM operates at a speed of $130 \mathrm{Mbps}$. That means there can be $0.37 \mathrm{M}$ cells switching through a switch within one second. If we use DES cipher with block size of 64 bits, then about 2M DES cipher blocks will go through a switch per second. If the number of VCs running through this switch is not too big, then with such an amount data, an attacker can easily break the session key in a relatively short time. Notice that always assuming that the hacker can not access such a computing power is not true in a short future, or even now. To avoid this kind of attack, the system has to change the session key frequently. If we assume that one session key can not be used more than 100M cipher blocks, then the lifetime of a session key becomes as short as several hundred seconds. This will make a lot of traditional key exchange mechanism inadequate. Even we have a scheme, which can successfully change the session key at this speed that will introduce another problem. In traditional key exchange mechanism, session key will be encrypted by a permanent key. Frequently exchanging session key will give enough information to an attacker to break the permanent key in a relative short amount of time.

As suggested in [ATMForum97], security will be applied on a per VC basis. That is to say, an encryptor/decryptor will use different keys for different VC. One advantage of this method is to protect the confidentiality of other $\mathrm{VC}$ in case one $\mathrm{VC}$ has been compromised. Another advantage the life time of session key can be reasonable long since normally the traffic of one VC is not so high as the total traffic of the system (this assumption might not be true in the future if multimedia becomes popular over the ATM). With this method, the system can even provide different quality of security service to different VC, therefore to introduce the concept of QoS into the ATM security.

However, one difficulty to implement this scheme in ATM is that it requires the "encryptor" to have the ability to access a range of key data at a high speed [TayFin95]. It also requires the "encryptor" can change the session key dynamically and apply to next cell very quickly. This requirement, called key agility, is non-trivial. As mentioned in [Eberle92], some cryptographic algorithms require a long set up time when the 
encrypted key changes. Even worse, according to large number of potential VCs, looking up the key in big key table will introduce a time overhead which can be in the critical path therefore can be the bottleneck of the system.

\section{CONCLUSIONS}

ATM technology perhaps is the most complex networking technology we ever have. To secure such a complex system will be even more difficult than design it. And now people just begin to discuss some issues of ATM security. It will take time for us to figure out how to completely achieve our security objectives. Because the goal of ATM is to provide a unified networking platform and communication infrastructure, ATM security, as part of this infrastructure, has to be flexible and compatible with other technology. That will introduce more difficulties to ATM security.

As we have discussed in this paper, there is a lot of research work been done on ATM security. Here, we present and compare solutions for ATM security proposed by Deng et al, Stevenson et al, Chuang, and ATM Forum. The ATM Forum Security Working Group has come up with drafts for security specifications and security framework which meets both operator's and user's security needs. On the other hand, the solutions proposed by Chuang, Deng, and Stevenson aim to ensure the end-to-end communication security over an ATM network considered as unsecured, thus, they focus on the user's security needs

The ATM Forum's solution is the most complete one in terms of security services offered (see Table 1), parameters possible to negotiate and implementation details level obtained (message structure) It also offers the biggest choices in terms of methods to be used for security services negotiation and renegotiations. However, this solution is also the most complicated one in terms of implementation.

Although, much research work has been done in the past few year on ATM security, more work need to be done regarding ATM firewalls, which means the methods of connecting public networks with public or private ATM networks. It is not yet clear however, just how, or whatever, it might be possible to implement firewalls in ATM environment. An other open issue is if and where a cryptographic mechanism should be added in the ATM signalling. Finally, there are some open issues which has to do with the ATM's capacity to support multi service traffic, like how to charge these 
services, how to maintain a secure billing system, how to combine a billing system with the electronic monetary systems.

\section{References}

[Alles95] Alles A., ATM Internetworking ,Presented at Engineering InterOp, Las Vegas, 1995

[ATMForum94] ATM Forum Technical Committee, ATMForum B-ICI specification, Version 1.1, 1994

[ATMForum95] ATM Forum Technical Committee, ATMForum B-ICI specification, Version 2.0, 1995

[ATMForum97] ATM Forum, Security Working Group, (Draft), Phase I ATM Security Specification, 1997

[ATMForum98] ATM Forum, Technical Committee, ATM Security Framework 1.0, 1998

[Chuang96] Chuang Shaw-Cheng, Securing ATM Networks, 3rd ACM Conference on Computer and Communications Security, pp. 19-30, New Delhi, India, 1996

[Deng95] Deng R. et al, Securing Data Transfer in Asynchronous Transfer Mode Networks, Proceedings of GLOBECOM'95, Singapore, pp. 1198-1202, 1995

[Eberle92] Eberle H., A High Speed DES Implementation for Network Applications, Advances in Cryptology-CRYPTO 92, Berlin Spring-Verlay, pp. 527-545, 1993

[GriKa96] Gritzalis D., Katsikas S., Towards a formal system-to-system authentication protocol, Computer Communications, Vol.19, pp.954-961, Elsevier Advanced Technology Publishers, 1996.

[Hanson95] Hanson L., The Impact of ATM on Security in Data Network, Proceedings of Compsec International 1995, Conf. 12, pp 318-324, 1995

[I.321] CCITT I.321, B-ISDN Protocol Reference Model and its Applications, 1991

[SteHilBy95] Stevenson D., Hillery N. and Byrd G., Secure Communications in ATM Networks, Communications of the ACM, Vol. 38, No.2, 1995.

[TayFin95] Taylor R., Findlow G., Asynchronous Transfer Mode: Security Issues, Proc. Australian Telecommunication Networks and Applications Conference, pp. 161-166, 1995 